\title{
COVID-19 infection in pediatric patients treated for cancer
}

\author{
Lital Oz-Alcalay ${ }^{1,3} \cdot$ Sarah Elitzur $^{2,3} \cdot$ Nofar Amitai $^{1,3} \cdot$ Helen Toledano $^{2,3} \cdot$ Shlomit Barzilai-Birenboim ${ }^{2,3}$. \\ Gali Avrahami $^{2,3} \cdot$ Gil Gilad $^{2,3} \cdot$ Yoav Vardi $^{2,4} \cdot$ Michal Dvori $^{2,3} \cdot$ Shai Izraeli $^{2,3} \cdot$ Gilat Livni $^{1,3} \cdot$ Oded Gilad $^{2,3}$ (])
}

Received: 6 September 2021 / Accepted: 30 October 2021 / Published online: 6 November 2021

(c) The Author(s) under exclusive licence to Japan Society of Clinical Oncology 2021, corrected publication 2021

\begin{abstract}
Background COVID-19, the novel coronavirus, has caused a global pandemic affecting millions of people around the world. Risk factors for critical disease in adults are advanced age and underlying medical comorbidities, including cancer. Data are sparse on the effect of COVID-19 infection on pediatric patients with cancer during their active antineoplastic therapy. The optimal management of antineoplastic treatment during COVID-19 infection in this unique population is controversial. Aim To describe the severity and clinical course of COVID-19 infection in pediatric patients with cancer during active antineoplastic treatment and to study their course of treatment.

Methods Clinical and laboratory data were collected from medical files of patients diagnosed with COVID-19, confirmed by polymerase chain reaction (PCR), who received active antineoplastic treatment between March 2020 and May 2021 in a large tertiary pediatric medical center.

Results Eighteen patients with diverse pediatric cancers are described. They were infected with COVID-19 at different stages of their antineoplastic treatment regimen. Eight had an asymptomatic COVID-19 infection, nine had mild symptoms, and one had severe disease. All of them recovered from COVID-19 infection. Two patients experienced delays in their antineoplastic treatment; none of the other patients had delays or interruptions, including patients who were symptomatic for COVID-19. Conclusion In pediatric patients with cancer who test positive for COVID-19, yet are asymptomatic or have mild symptoms, the continuance of antineoplastic therapy may be considered.
\end{abstract}

Keywords COVID-19 $\cdot$ Pediatric patients $\cdot$ Cancer $\cdot$ Antineoplastic therapy

\section{Introduction}

At the end of 2019, a novel coronavirus (COVID-19) was identified as the cause of a cluster of respiratory diseases resulting in an epidemic throughout the world. The virus is designated severe acute respiratory syndrome coronavirus 2 (SARS-CoV-2) [1]. The clinical spectrum of SARS-CoV-2

OG and GL contributed equally to this work.

Oded Gilad

dkgsguss@gmail.com

1 Department A, Hematology-Oncology Schneider Children's Medical Center of Israel, Petach Tikva, Israel

2 Department of Hematology-Oncology, Schneider Children's Medical Center of Israel, Petach Tikva, Israel

3 Sackler School of Medicine, Tel Aviv University, Tel Aviv, Israel

4 Department B, Hematology-Oncology Schneider Children's Medical Center of Israel, Petach Tikva, Israel infection ranges from asymptomatic infection, through mild respiratory symptoms to severe disease, presenting with dyspnea and hypoxia, and a potential deterioration to respiratory failure, shock, multi-organ dysfunction, and fatal illness [2]. The most critical or fatal disease occurs predominantly in hospitalized adults with advanced age or with certain underlying medical comorbidities including cancer [3-6]. Adult oncological patients infected with COVID19 often have severe disease [7-9]. However, in pediatric patients with cancer, the impact of chemotherapy administration at the time of COVID-19 infection is yet to be determined. Due to their immunocompromised state, children undergoing cancer-directed therapy have been surmised to be at a higher risk for COVID-19-associated complications and fatalities; however, data to support this are lacking [10].

Here, we present a series of eighteen pediatric patients with COVID-19 infection during active antineoplastic treatment for diverse diagnoses in a tertiary medical center in Israel. Our data suggest that chemotherapy may be compatible with COVID-19 infection in pediatric oncology patients. 


\section{Methods}

We surveyed all pediatric patients treated for cancer from March 2020 to May 2021 in the largest tertiary pediatric medical center in Israel. The diagnosis of SARS-CoV-2 was based on a positive polymerase chain reaction (PCR) test from a nasopharyngeal swab, regardless of symptoms. Of note, in accordance with institutional policy, all admitted patients were routinely tested for SARS-CoV-2 during this period, regardless of the reason for their hospitalization. Data were collected from patients' medical files, including clinical and laboratory parameters. COVID-19 was categorized according to the US National Institutes of Health (NIH) classification severity index: asymptomatic, mild, moderate, or severe disease [11].

The study protocol was approved by our institutional review board (0914-20-RMS).

\section{Results}

Eighteen pediatric patients with cancer who tested positive for SARS-CoV-2 were admitted for antineoplastic treatment during the study period: eight females and ten males. They represent $6.4 \%$ of the 280 children who were newly diagnosed and treated for cancer in our medical center during the 14-month study period. The median age at SARS-CoV-2 diagnosis was 11.7 years (range $1.8-18$ years) (Table 1 ). The most common underlying malignancy was acute lymphoblastic leukemia $(n=10 ; 56 \%)$. Other underlying malignancies included Burkitt lymphoma $(n=2)$, low-grade glioma $(n=2)$, Hodgkin lymphoma $(n=1)$, Ewing sarcoma $(n=1)$, high-grade glioma $(n=1)$, and Langerhans cell histiocytosis $(n=1)$. Twelve patients were diagnosed with COVID19 while receiving intravenous chemotherapy (one was also receiving radiotherapy), four were diagnosed during maintenance therapy for acute lymphoblastic leukemia (oral methotrexate/mercaptopurine), one patient was diagnosed while receiving combined oral chemotherapy and biological agents (BRAF inhibitors) and one patient was receiving radiotherapy only (Table 1). None of the patients had received the SARS-CoV-2 vaccine, which had not yet been approved for the pediatric population at the time of our study.

COVID-19 infection was classified as asymptomatic in eight patients (44\%) and mild in nine (50\%); only one patient had severe disease, with a severe respiratory presentation (described below). Presenting symptoms included fever in six patients (33\%) and rhinorrhea in three (17\%); Only one patient had significant respiratory distress secondary to COVID-19 infection; all the other patients had normal oxygen saturations and did not require oxygen support.
All the patients were admitted to isolated rooms designated for the treatment of COVID-19 patients. The median length of hospital stay was three days (range 1-40). The duration of hospitalization was not extended for any of the patients due to the COVID19- infection; the patients remained in the hospital as required for the administration of their chemotherapeutic regimens. Three patients were treated with antibiotics due to fever at presentation, discontinued upon receiving negative culture results (Table 1). Six patients were not admitted to the hospital and continued treatment as outpatients. None of the patients received COVID-19-specific therapy. The median duration of COVID-19 PCR positivity among hospitalized patients was 40 days (range 9-130 days). None of the patients experienced antineoplastic treatment delays due to COVID-19 infection.

A negative correlation was demonstrated between lymphocyte counts and the duration of COVID-19 PCR positivity, with a trend towards statistical significance. The mean lymphocyte count of 11 patients, treated with active intravenous chemotherapy, was $0.66 \pm 0.48 \times 10^{9} / \mathrm{L}$ and the meantime to negative PCR was $52 \pm 42.6$ days. This compared to $1.44 \pm 1.16 \times 10^{9} / \mathrm{L}$ and $21.9 \pm 7.6$ days for those who received oral chemotherapy or other treatment $(P=0.085)$ (Fig. 1).

The single incidence of severe COVID-19 occurred in a 15-year-old female (patient number 13, Table 1). She was admitted to the intensive care unit with shortness of breath and low blood pressure. Emergency electrocardiogram and echocardiography performed in the resuscitation room upon arrival demonstrated severe pericardial and pleural effusion. Emergency pericardiocentesis was performed, with drainage of $900 \mathrm{ml}$ of serous fluid and immediate clinical improvement. COVID-19 infection was diagnosed following routine PCR examination at admission. Abdominal and chest CT scan demonstrated a mediastinal mass with superior vena cava syndrome, main bronchus stenosis, and pericardial effusion. Pericardial effusion analysis established a diagnosis of Burkitt lymphoma. When stabilized, the patient was transferred to a department dedicated to treating patients with COVID-19, where she received two cycles of induction chemotherapy (based on Inter-B-NHL Ritux 2010 protocol [12], NCT01516580) and remained an inpatient for 40 days. During her hospital stay, she was respiratory stable; her lowest saturation index was $94 \%$, with no need of oxygen support. Her COVID-19 infection was classified as severe due to severe grunting and dyspnea upon admission. Throughout her hospitalization, she remained COVID-19 positive, as confirmed by both PCR and cell culture. 


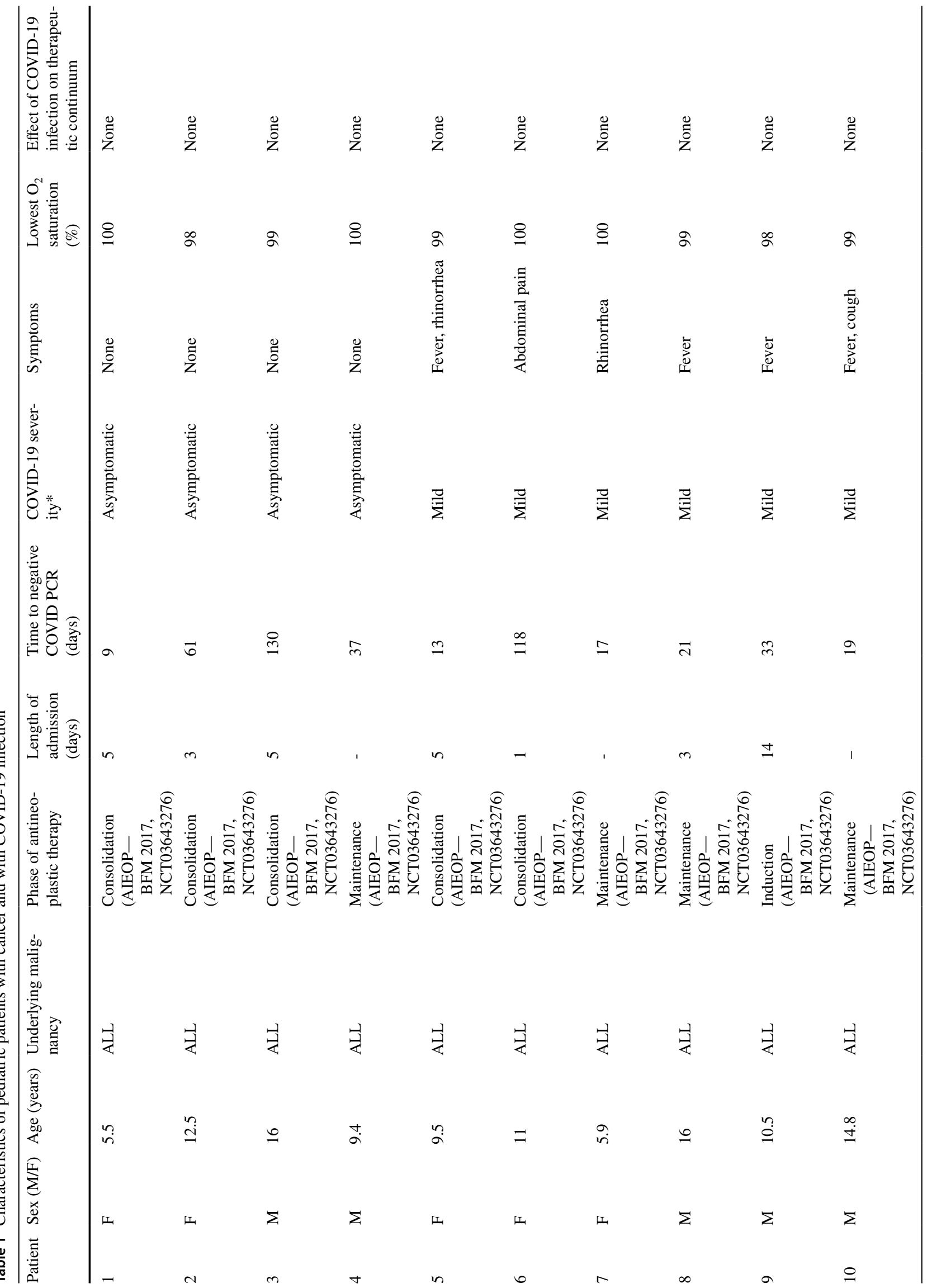




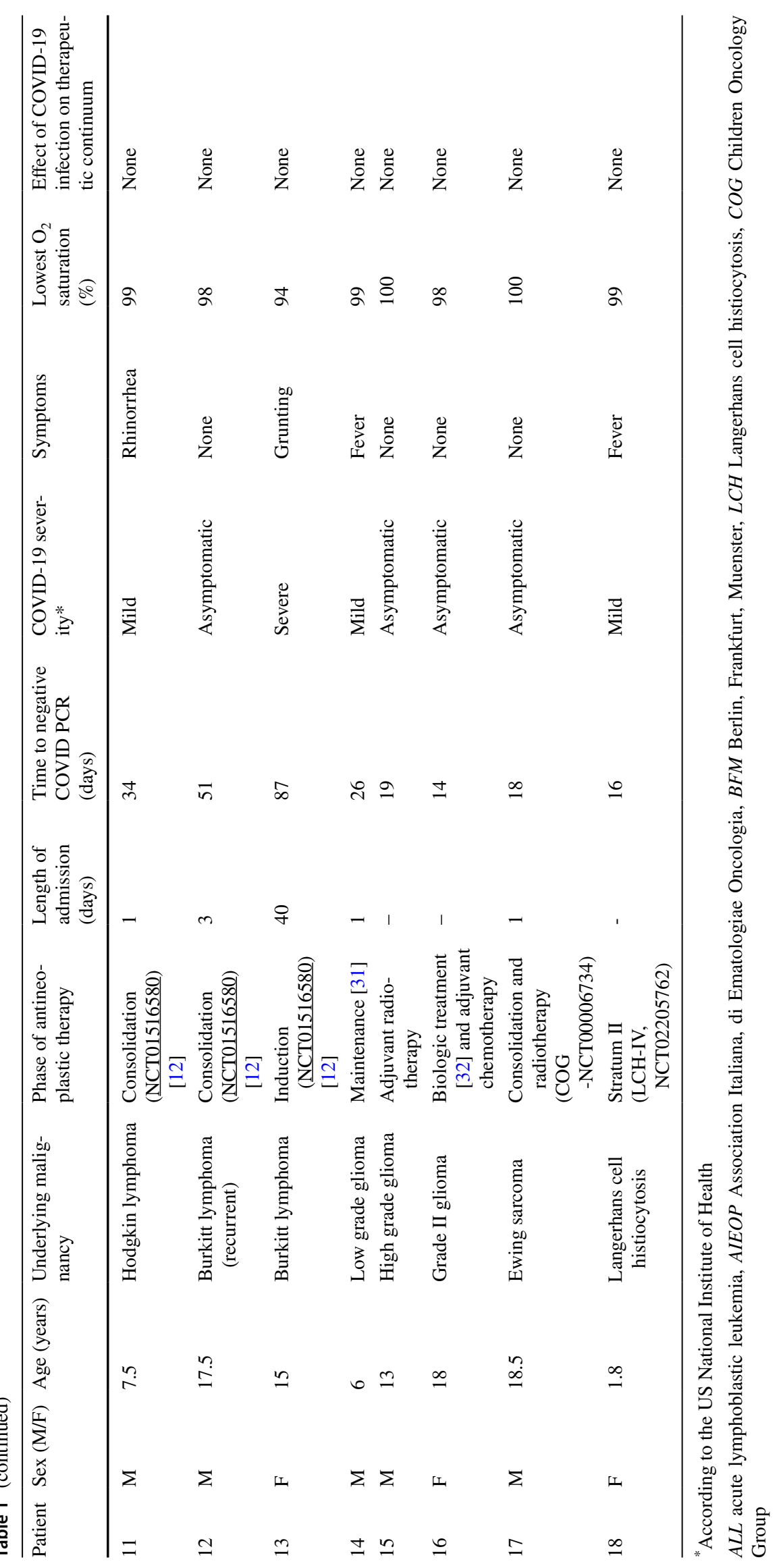




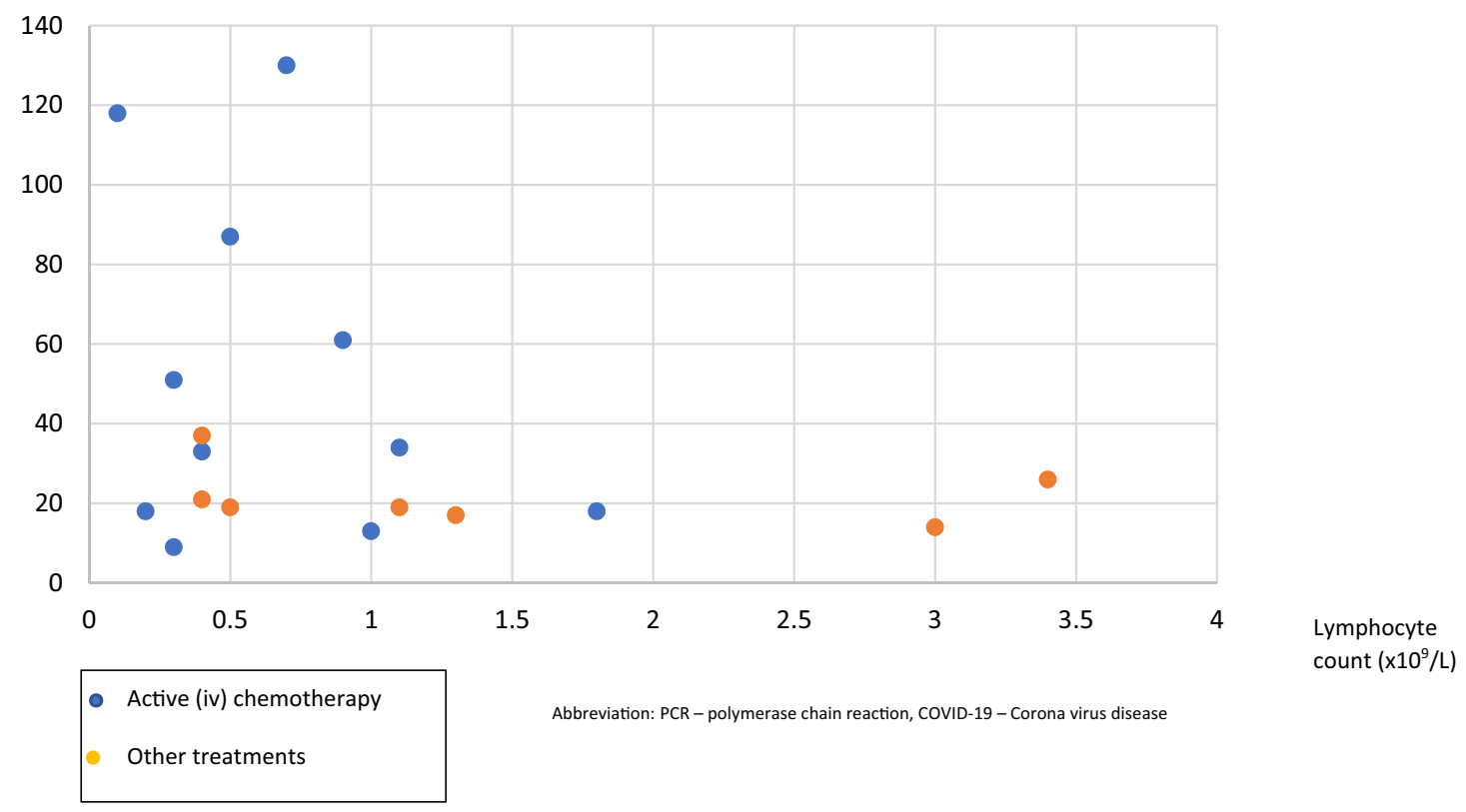

Fig. 1 The duration of positive COVID-19 PCR according to lymphocyte count, in pediatric patients treated for cancer. Each dot represents the data of a single patient. Seven were treated with active intra-

\section{Discussion}

We report a series of 18 pediatric oncological patients who were positive for COVID-19 at our center during the period of March 2020-May 2021. We found that COVID19 infection had little or no impact on the course of active antineoplastic treatment and the therapeutic continuum in our patients. Active antineoplastic treatment did not have any apparent effect on COVID-19 severity status or the development of chemotherapy complications in our patients with COVID- 19 .

Several reports have concluded that adults with cancer are particularly vulnerable to SARS-CoV-2 infection, and especially to severe COVID-19. This is evident by an increased number of hospital admissions, more admissions to the intensive care unit, and a greater need for supplemental oxygen and mechanical ventilation. Among adults, independent risk factors identified for severe COVID-19 infection include increased age, male sex, and chronic comorbidities, including obesity and cancer $[3,4,7]$. In adults with cancer, smoking status, multiple comorbidities, low-performance status, and active cancer treatment have been associated with severe COVID-19 infection [8, 13]. Adults with cancer have also tended to develop COVID19-related complications [8] and to have poorer outcomes than the general population [13, 14], including increased 30-day all-cause mortality [7].

Children were found to be less affected by SARS-CoV-2 than adults, and account for $1-8 \%$ of confirmed incidences venous chemotherapy (yellow dots) and 11 received other treatments (blue dots). PCR polymerase chain reaction, COVID-19 Coronavirus disease

of COVID-19. When affected, children usually have a milder disease [11, 13-16].

Several previous reports have suggested that pediatric patients with cancer, infected with COVID-19, are at an increased risk of severe disease due to their underlying malignancy and treatment effect, similar to adults with cancer [16]. Pediatric patients with cancer who test positively for COVID-19 have been reported to require intensive care admission and to have unfavorable outcomes [17, 18]. Continued chemotherapy during COVID-19 infection has been reported to result in dismal outcomes $[17,18]$.

In contrast to the above, others have argued that COVID-19 has minimal effect on pediatric cancer patients, and that those affected are mostly asymptomatic or with only mild disease [19-21]. Madhusoodhan et al. [10], who described pediatric patients with cancer during active therapy, concluded that they appear to have a higher risk of severe disease and a greater need of critical care support compared to the general pediatric population. However, this risk may be lower than initially perceived and is far lower than that observed in adult oncology patients. Similarly, in their cohort of pediatric hematology and oncology patients, Gampel et al. [22] described relatively mild COVID-19 (male patients had a slightly more severe clinical course). The children were treated as outpatients, without the need for respiratory support. In light of the uncertainties regarding the impact of COVID-19 on oncology patients, delays have been commonly reported: in cancer diagnoses, due to fear of exposure by patients, parents, 
and physicians; and in therapy of diagnosed patients [10, 22-24].

The majority of our patients with cancer who were positive for COVID-19 had underlying hematological malignancies and were diagnosed with COVID-19 during the induction or consolidation phases of therapy, which consist of intensive antineoplastic treatment.

We noticed a negative correlation between lymphocyte counts and the duration of COVID-19 PCR positivity. However, in several patients, the duration of COVID19 PCR positivity was shorter than 20 days, despite low $\left(<0.5 \times 10^{9} / \mathrm{L}\right)$ lymphocyte counts. Evidently, other factors contribute to the duration of COVID-19 PCR positivity.

Only one patient was considered to have a severe COVID-19 infection (US NIH classification severity index [11]), based on dyspnea and desaturation at presentation. We suspect that this patient's extensive mediastinal mass was the main cause of her symptoms, rather than COVID19, and that none of our patients actually had COVID-19 disease more severe than mild. In two patients, chemotherapy was delayed due to fever, which was attributed to either COVID-19 infection or to another viral infection.

Adherence to chemotherapeutic protocols and receiving treatment in due time is of utmost importance for the overall prognosis of pediatric cancers. This explains overall prognosis of pediatric cancers. This explains the treating oncologists' decisions in all the described patients to continue antineoplastic treatment regimens, generally without delay. All the patients received their intended treatment without complications secondary to either antineoplastic treatment or COVID-19 infection.

Interestingly, the median duration of positive COVID19 PCR in our cohort was 40 days (range 9-130 days). In comparison, median durations between 8 and 19.5 days have been reported for viral shedding of respiratory specimens in several papers on pediatric populations [25-27]. The difference may be explained by our patients' depressed immune system, resulting from their underlying malignancies and the antineoplastic treatment they received. Notably, in patients with low lymphocyte counts, the duration of SARS-CoV-2 PCR positivity was longer, as described earlier $[28,29]$. Nonetheless, most of our patients had asymptomatic or mild disease, and all recovered from COVID-19. Positive COVID-19 viral PCR is an important tool for diagnosing COVID-19; however, our results support the view that treatment should not be delayed until attaining PCR negativity $[15,24,30]$.

Our study has several limitations, such as a small sample size, with a preponderance for hematological malignancies. Only two patients were receiving induction therapy at the time of COVID-19 diagnosis. Nevertheless, our work provides an important representation of the pediatric hematology-oncology population during the COVID-19 era and provides valuable data regarding their treatment.

\section{Conclusions}

Our pediatric patients with cancer who were positive for COVID-19 but were asymptomatic or had mild COVID-19 symptoms did not experience exacerbation of COVID-19 symptoms as a result of active antineoplastic treatment. Our findings suggest that uninterrupted continuance of antineoplastic therapy may be considered in pediatric patients with cancer who are COVID-19-positive, and asymptomatic or with mild COVID-19 symptoms.

Acknowledgements Funding from the Israel Children's Cancer Foundation (New York), Israel Cancer Association, Hayim Association, and Larger than Life Foundation.

\section{References}

1. World Health Organization. World Health Organization. DirectorGeneral's remarks at the media briefing on 2019-nCoV [Internet]. http://www.who.int/dg/speeches/detail/who-director-gener al-s-remarks-at-the-media-briefing-on-2019-ncov-on-11-febru ary-2020 (Accessed on February 12, 2020) [cited 2021 Jun 17] Available from: http://www.who.int/dg/speeches/detail/who-direc tor-general-s-remarks-at-the-media-briefing-on-2019-ncov-on-11february-2020 (Accessed on February 12, 2020).

2. Wu Z, McGoogan JM (2020) Characteristics of and important lessons from the coronavirus disease 2019 (COVID-19) Outbreak in China: summary of a report of 72314 cases from the chinese center for disease control and prevention. JAMA 323:1239-1242

3. Centers for Disease Control and Prevention. Centers for Disease Control and Prevention. Science brief: Evidence used to update the list of underlyingmedical conditions that increase a person's risk of severe illness fromCOVID-19. Accessed on April 5, 2021 [Internet][cited 2021 Jun 17] https://www.cdc.gov/coronavirus/ 2019-ncov/science/science-briefs/underlying-evidence-table.html

4. Centers for Disease Controland Prevention. Centers for Disease Controland Prevention. Underlying Medical Conditions Associated with High Risk forSevere COVID-19: Information for Healthcare Providers [Internet][cited 2021 Jun 17] Available from: https://www.cdc.gov/coronavirus/2019-ncov/hcp/clinicalcare/underlyingconditions.html

5. Rosenthal N, Cao Z, Gundrum J, et al. Risk Factors Associated With In-Hospital Mortality in a US National Sample of Patients With COVID-19. JAMA Netw Open, 2020 3: e2029058.

6. Williamson EJ, Walker AJ, Bhaskaran K et al (2020) Factors associated with COVID-19-related death using OpenSAFELY. Nature 584:430-436

7. Kuderer NM, Choueiri TK, Shah DP et al (2020) Clinical impact of COVID-19 on patients with cancer (CCC19): a cohort study. Lancet 395:1907-1918

8. Liang W, Guan W, Chen R et al (2020) Cancer patients in SARSCoV-2 infection: a nationwide analysis in China. Lancet Oncol 21:335-337

9. Onder G, Rezza G, Brusaferro S (2020) Case-fatality rate and characteristics of patients dying in relation to COVID-19 in Italy. JAMA 323:1775-1776 
10. Madhusoodhan PP, Pierro J, Musante J, et al. Characterization of COVID-19 disease in pediatric oncology patients: The New YorkNew Jersey regional experience. Pediatr Blood Cancer, 2021 68: e28843.

11. N I H. Clinical Spectrum of SARS-CoV-2 Infection, NIH Last Updated: April 21, 2021 [Internet][cited 2021 Jun 17] https:// www.covid19treatmentguidelines.nih.gov/overview/clinical-spect rum/

12. Minard-Colin V, Aupérin A, Pillon M et al (2020) Rituximab for high-risk, mature B-cell non-hodgkin's lymphoma in children. $\mathrm{N}$ Engl J Med 382:2207-2219

13. Dai M, Liu D, Liu M et al (2020) Patients with cancer appear more vulnerable to SARS-CoV-2: a multicenter study during the COVID-19 outbreak. Cancer Discov 10:783-791

14. Kim L, Whitaker M, O'Halloran A et al (2020) Hospitalization rates and characteristics of children Aged $<18$ years hospitalized with laboratory-confirmed COVID-19 - COVID-NET, 14 States, March 1-July 25, 2020. MMWR Morb Mortal Wkly Rep 69:1081-1088

15. Posfay-Barbe KM, Wagner N, Gauthey M, et al. COVID-19 in Children and the Dynamics of Infection in Families. Pediatrics , 2020146.

16. Docherty AB, Harrison EM, Green CA, et al. Features of 20133 UK patients in hospital with covid-19 using the ISARIC WHO Clinical Characterisation Protocol: prospective observational cohort study. BMJ , 2020 369: m1985.

17. Stokes CL, Patel PA, Sabnis HS, et al. Severe COVID-19 disease in two pediatric oncology patients. Pediatr Blood Cancer, 2020 67: e28432.

18. Fonseca EV, Pardo CA, Linares A et al (2021) Clinical characteristics and outcomes of a cohort of pediatric oncohematologic patients with COVID-19 infection in the City of Bogotá. Colombia Pediatr Infect Dis J 40:499-502

19. Hrusak O, Kalina T, Wolf J et al (2020) Flash survey on severe acute respiratory syndrome coronavirus- 2 infections in paediatric patients on anticancer treatment. Eur J Cancer 132:11-16

20. Wolfs TFW, Attarbaschi A, Balduzzi A, et al. COVID-19-Impact on Childhood Haematology Patients. HemaSphere, 2020 4: e465.

21. Hamdy R, El-Mahallawy H, Ebeid E. COVID-19 infection in febrile neutropenic pediatric hematology oncology patients. Pediatr Blood Cancer , 2021 68: e28765.

22. Gampel B, Troullioud Lucas AG, Broglie L, et al. COVID-19 disease in New York City pediatric hematology and oncology patients. Pediatr Blood Cancer, 2020 67: e28420.
23. Dvori M, Elitzur S, Barg A, et al. Delayed diagnosis andtreatment of children with cancer during the COVID-19 pandemic. Int J Clin Oncol

24. Weissleder R, Lee H, Ko J, et al. COVID-19 diagnostics in context. Sci Transl Med, 202012.

25. Bahar B, Jacquot C, Mo YD et al (2020) Kinetics of viral clearance and antibody production across age groups in children with severe acute respiratory syndrome coronavirus 2 infection. J Pediatr 227:31-37.e1

26. Lu Y, Li Y, Deng W et al (2020) Symptomatic infection is associated with prolonged duration of viral shedding in mild coronavirus disease 2019: a retrospective study of 110 children in Wuhan. Pediatr Infect Dis J 39:e95-e99

27. De Ioris MA, Scarselli A, Ciofi Degli Atti ML, et al. Dynamic Viral Severe Acute Respiratory Syndrome Coronavirus 2 RNA Shedding in Children: Preliminary Data and Clinical Consideration from a Italian Regional Center. J Pediatric Infect Dis Soc, 2020 9: 366-369.

28. Gautret P, Million M, Jarrot PA et al (2020) Natural history of COVID-19 and therapeutic options. Expert Rev Clin Immunol 16(12):1159-1184. https://doi.org/10.1080/1744666X.2021. 1847640 (Epub 2020 Dec 24)

29. Cheng MP, Papenburg J, Desjardins M et al (2020) Diagnostic testing for severe acute respiratory syndrome-related coronavirus 2: a narrative review. Ann Intern Med 172:726-734

30. Terpos E, Ntanasis-Stathopoulos I, Elalamy I et al (2020) Hematological findings and complications of COVID-19. Am J Hematol 95:834-847

31. Packer RJ, Ater J, Allen J et al (1997) Carboplatin and vincristine chemotherapy for children with newly diagnosed progressive lowgrade gliomas. J Neurosurg 86:747-754

32. Nobre L, Zapotocky M, Ramaswamy V, et al. Outcomes of BRAF V600E Pediatric Gliomas Treated with Targeted BRAF Inhibition. JCO Precis Oncol. 2020 May 20;4:PO.19.00298. https://doi.org/ 10.1200/PO.19.00298. PMID: 32923898; PMCID: PMC7446502.

Publisher's Note Springer Nature remains neutral with regard to jurisdictional claims in published maps and institutional affiliations. 